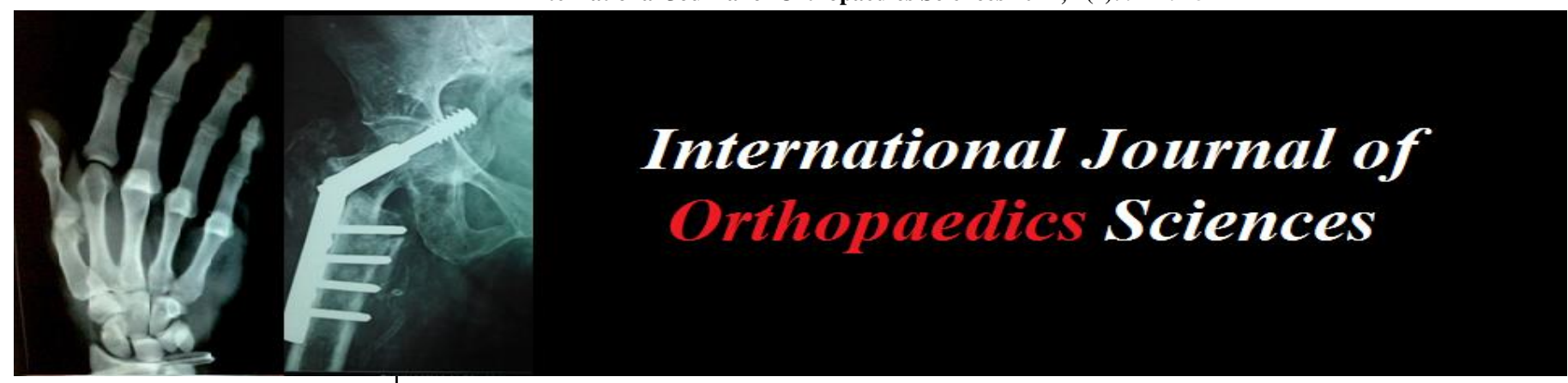

E-ISSN: 2395-1958

P-ISSN: 2706-6630

IJOS 2021; 7(1): 921-925

(C) 2021 IJOS

www.orthopaper.com

Received: 19-11-2020

Accepted: 21-12-2020

Dr. Shailendra Chauhan MBBS MS (Orthopaedics)

Assistant Professor,

Department of Orthopaedics

Govt. Medical College Khandwa,

Madhya Pradesh, India

Dr. Sunil Kumar Dhanger

Senior Resident,

Department of Orthopaedics

Govt. Medical College Khandwa,

Madhya Pradesh, India

Dr. Bhanu Pratap Singh

Senior Resident,

Department of Orthopaedics

Govt. Medical College Khandwa,

Madhya Pradesh, India
Corresponding Author: Dr. Shailendra Chauhan MBBS MS (Orthopaedics), Assistant Professor,

Department of Orthopaedics Govt. Medical College Khandwa, Madhya Pradesh, India

\section{Study of surgical management of distal femur fracture using locking compression plate}

\author{
Dr. Shailendra Chauhan, Dr. Sunil Kumar Dhanger and Dr. Bhanu \\ Pratap Singh
}

DOI: https://doi.org/10.22271/ortho.2021.v7.i1n.2589

\begin{abstract}
Introduction: Distal femur fracture are being treated with distal femur nail, dynamic condylar screw and even addition of a medial plate with good clinical results. By this study, we explore the potenti ability of a distal femur locking plate to counter distal femur fractures of extra articular, partial or intra- articular nature. Positive results have been brought out by various groups from all over the world.

Aim: To study the clinical, functional and radiological outcome of distal femoral fractures managed by open reduction and internal fixation with distal femur locking plate in skeletally mature patients.

Materials and Methods: This was a prospective study conducted from January 2018 to January 2021 at the Government Medical College Khandwa with a 6month follow-up in skeletally mature patients with post-traumatic distal femur fractures were included. Follow and evaluation was done according to the Neer scoring system. The statistical data analysis was carried out using SPSS version 20 (IBM, Chicago, USA). The $\mathrm{p}$-value $<0.05$ was considered significant.

Results: Following all principles of fracture reduction, union was achieved in all patients with mean time to radiological union being 19 weeks. The mean Range of Motion (ROM) was 109 degrees with 20 patients having a Neer score graded as excellent to satisfactory. Three patients developed complications in the form of infection (two cases) and mal-union (one case) during the course of our study, but were completely treated by the end of the study.

Conclusion: Positive results can be obtained by distal femur locking plate alone as it is the main implant of choice for distal femur fractures of all varieties. Best outcome is expected if fracture fixation is done following all the basic principles of fracture fixation and taking benefit of the mechanical properties of a locking plate.
\end{abstract}

Keywords: surgical management, distal femur fracture, locking compression plate

\section{Introduction}

Distal femoral fractures account for $4-7 \%$ of all femoral fractures. ${ }^{1}$ The distal femoral fracture occurs in approximately 37 per $1,00,000$ per years. ${ }^{2,3,4}$ This fracture occurs in a particular bimodal distribution, $1^{\text {st }}$ group patients are below 40 years of age, mostly males, sustained high energy trauma such as RTA or a fall from heights; second group consists of patients above 50 years, mostly females, with osteoporosis sustained relatively low energy trauma ${ }^{[5-8]}$. These fractures are difficult to treat because of osteoporosis, severe comminution, too short distal femoral fragment ${ }^{[9]}$ compound injuries and associated ligament injuries, meniscal and extensor mechanism injuries ${ }^{[1]}$.

The goals of surgical treatment should be anatomic reduction of the articular surface, restoration of limb alignment, length, and rotation, bone grafting for extensive bone loss and stable fixation that allows for early mobilization ${ }^{[10]}$ After the development of various implants and techniques, the management of distal femoral fractures has shifted from non-operative to operative methods ${ }^{[11,12]}$. Operative methods include traditional plating technique that require compression of the implant to the femoral shaft (blade plate, dynamic condylar screw, nonlocking condylar buttress plate), antegrade/retrograde nail fixation, submuscular locked internal fixation and external fixation ${ }^{[2,13-15]}$. Distal femoral locking compression plate allows both locking and compression screw fixation of the distal femur fracture along with articular surface realignment ${ }^{[10]}$. It works on the principle of rigid fixation [2, 16-18], acts as an extramedullary load bearing device ${ }^{[5,19]}$ leading to early union along with knee mobilization. 
It works on splinting rather than compression, therefore it provides flexible stabilization causes induction of callus formation and it avoids stress ${ }^{[2,20,21]}$. Recent generation of distal femoral locking compression plate is precontoured plate based on the anatomy of bone. The pull out strength of locked screws better than conventional screws and that's why, it is useful in osteoporotic bone ${ }^{[22]}$.

The purpose of this study is to evaluate functional outcome, fracture healing and the complications of distal femoral fractures using open reduction and internal fixation with distal femoral locking compression plate.

\section{Material and Methods}

This was a prospective study conducted from January 2018 to January 2021 at Government Medical College Khandwa with a 6 month follow-up. The study was approved by Institutional Ethical Committee and informed consent was taken from all the 37 patients included in the study.

Inclusion Criteria

1. All patients who are above the age of 18 years having distal femoral fractures.

2. Exclusion Criteria

3. Patients of age less than 18 years.

4. Open distal femur fractures.

5. Pathological fractures.

6. Associated tibia fractures.

7. Distal femur fractures with neuro-vascular compromise.

8. Patient having other fractures in the same limb, hip and vertebra.

Initial resuscitation of patients was done in emergency department, limb splinted, appropriate radiographs and CT scan as per requirements was done. Preoperative investigations were done consisting of haemoglobin level, platelet count, serum electrolytes and renal function tests, coagulogram, fasting or random blood sugar levels and viral markers consisting of Hepatitis B, C and HIV. A blood group cross-match of the patient was sent to the transfusion medicine department of the hospital prior to surgery. A chest $\mathrm{X}$-ray and ECG were done in those patients as required by the anaesthetic team. Spinal or epidural anaesthesia was given. Srubbing painting drapping done using standard protocols . Standard lateral approach by developing the plane between vastus lateralis muscle and lateral intermuscular septum was used for closed fractures of distal femur. Lateral parapatellar arthrotomy by using the Swash- buckler approach or its modifications were used to address the articular involvement of the lateral femoral condyle or the inter- condylar notch. A dual incision (standard lateral plus a medial subvastus incision) was also done in cases where a Hoffa's fracture or articular fracture of the medial femoral condyle had to be addressed in cases where access via the lateral approach was not possible. We always strived for obtaining anatomical reduction and good solid fixation in the articular area of the distal femur even at the cost of spending an extra amount of time, rather than giving the patient an incongruous joint. Distal femur locking condylar plate was used for fracture fixation using locking, $4.5 \mathrm{~mm}$ cortical screws and partiallythreaded cancellous screws of diameter $4.5 \mathrm{~mm}$, Herbert screws for smaller articular fragments and $6.5 \mathrm{~mm}$ for articular reduction of condyles. Technique of counter sinking for screws heads was used when proper seating of the plate had to be obtained. Length of the plate used was judged based on the extent of fracture of distal femur. In cases of fractures with metaphyseal-comminution with no bony reference point along the lateral cortex for direct fracture reduction the shaft of femur was aligned in the center of the condyles and it was ensured to prevent lateralization of the shaft at time of plate application Primary bone grafting was done in closed cases where the demand for such a procedure was gauged by the operating surgeon. The average blood loss was $300-400 \mathrm{ml}$ in each case. No use of tourniquets was done in any of the case. Post-operatively suction drain was removed after 24-48 hours and first wound inspection was done on 2rd post-operative day. Intra-venous antibiotics were continued for 24 hours in closed fractures. Post-operative physiotherapy regime was tailored according to the fracture pattern and fixation achieved. In cases where ever possible, knee bending and assisted knee Range of Motion (ROM) exercises were initiated on postoperative day 1. Patients were mobilized based on the degree of bone quality, severity of injuries, and pattern of fractures. At post-operative day 2 to 3, the patients were mobilized with crutches/walker until 6 weeks. Full weight bearing ambulation without any aids was started at approximately 3 months in majority of the cases with radiographic evidence of fracture union. Patients were discharged at post-operative day 3 with stitch removal being done on day $14^{\text {th }}$, making it convenient for the patient to take bath and maintain good body hygiene. Regular follow-up of all cases was done at 6 weeks, 12 weeks and 24 weeks. At each follow-up patients were evaluated clinically using knee society Neer's scoring system and radiologically with appropriate X-rays. No patients were lost to follow-up.

\section{Statistical analysis}

The statistical data analysis was carried out using a computer based statistical analysis programme, SPSS version 20 (IBM, Chicago, USA). For the statistical data analysis, paired t-test was used between two correlated groups while for uncorrelated groups; means were compared using independent t-test. A p-value

\section{Results}

Table 1: Distribution of Age of Distal Femur Fracture Patients

\begin{tabular}{|c|c|c|}
\hline Age (year) & Frequency (N) & Percent (\%) \\
\hline $20-30$ & 3 & 8.1 \\
\hline $30-40$ & 12 & 32.4 \\
\hline $40-50$ & 8 & 21.6 \\
\hline $50-60$ & 5 & 13.5 \\
\hline$\geq 60$ & 9 & 24.3 \\
\hline Total & 37 & 100 \\
\hline
\end{tabular}

Out of the 37 cases in our study, the mean age of cases in our study was $45.70 \pm 15.35$ years ranging from 20 to 78 years approximately $25(67.6 \%)$ of the patients were male, $12(32.4 \%)$ were female. The road traffic accident was the most common mode of injury, in 27 number (72.9\%). Fall due to various reasons experienced by 10 patients $(27.1 \%)$ The side affected in distal femur fracture among studied patients was noted more on left by $54.1 \%$ while the right seventeen $(45.9 \%)$ cases. 
Table 2: The frequency and percentage distribution of preoperative diagnosis to muller's classification

\begin{tabular}{|c|c|c|}
\hline Preoperative Diagnosis & Frequency (N) & Percent (\%) \\
\hline Muller Type A-1 & 10 & 27.0 \\
\hline Muller Type A-2 & 3 & 8.1 \\
\hline Muller Type A-3 & 5 & 13.5 \\
\hline Muller Type B-1 & 7 & 18.9 \\
\hline Muller Type B-2 & 2 & 5.4 \\
\hline Muller Type B-3 & 2 & 5.4 \\
\hline Muller Type C-1 & 4 & 10.8 \\
\hline Muller Type C-2 & 1 & 2.7 \\
\hline Muller Type C-3 & 3 & 8.1 \\
\hline
\end{tabular}

In our study, 6(16.2\%) patient had post-operative complication two patients had early post-operative complication in the form of superficial infection. These were then treated with culture sensitive parenteral antibiotics and antiseptic dressing, which resulted in satisfactory healing of the fracture. One case was found to have late complication in the form of mal-union of the distal femur fracture [Table 3]. Out of total, 37 patients, six patients (16\%) achieved full flexion of 130 degrees or more. Better outcome was observed in terms of range of motion at knee joint in extra-articular fractures $(82 \%)$ than intra- articular fractures $(72 \%)$ treated with distal femur locking plate. The mean ROM in all 37 patients was 114 degrees.

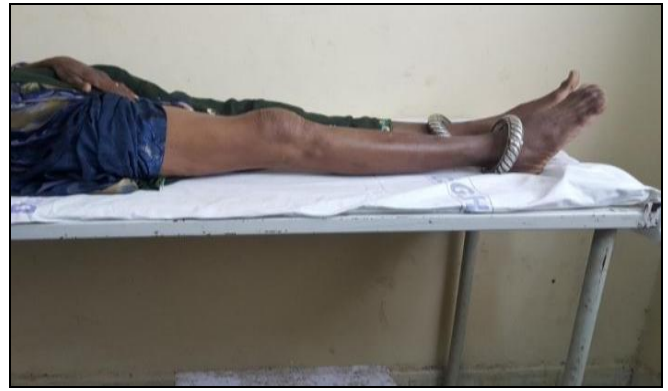

Fig 1: clinical photograph at 6 month followup

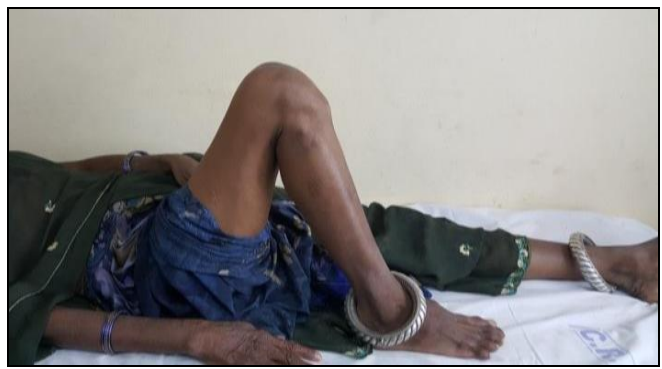

Fig 2: clinical photograph at 6 month follow up
Table 3: Distribution of late complications of distal femur fracture patients

\begin{tabular}{|c|c|c|}
\hline Late complications & Frequency (N) & Percent (\%) \\
\hline Wound infection & 2 & 5.4 \\
\hline Knee stiffness & 3 & 8.1 \\
\hline Malunion & 1 & 2.7 \\
\hline Total & 6 & 16.2 \\
\hline
\end{tabular}

According to Neer's criteria which included evaluation in terms of pain, range of motion, walking and work capacity, anatomy and X-ray findings score at 6 months was calculated results were compared in case of intra- and extra-articular fractures, clinically ROM at knee joint and score at 6 months was better in extra-articular fractures than intra-articular, although statistically insignificant ( $\mathrm{p}$-value $=0.5012$ ) in this situation as well. In the present study, results were tabulated into 4 groups i.e., excellent, satisfactory, unsatisfactory and poor according to criteria laid down by Neer et al., ${ }^{[11]}$, the results were excellent in $14(37.8 \%)$, good in $8(48.6 \%)$ fair in $3(8.1 \%)$ and poor in $2(5.4 \%)$ patients.
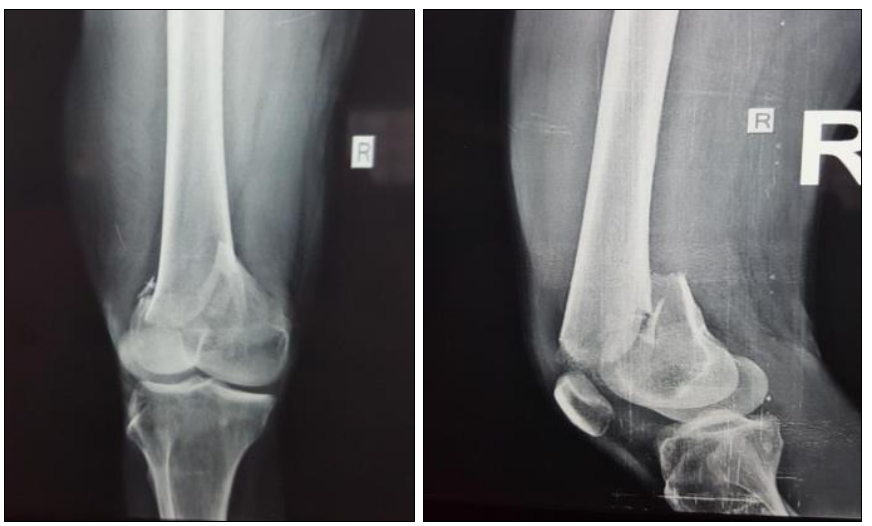

Fig 3: Pre-Operative X-ray Antero-Posterior view and lateral view
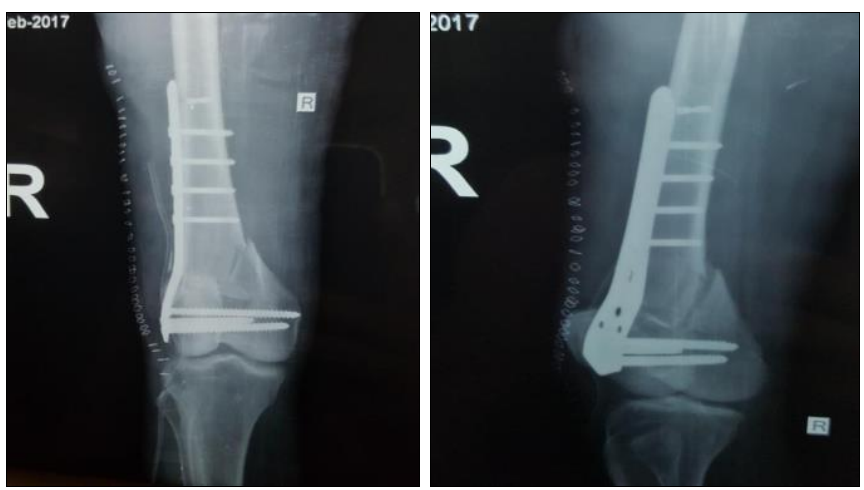

Fig 4: Post-operative $X$ ray Anteroposterior and Lateral view

Table 4: Assessment of clinical and functional outcome by neer's society scoring system

\begin{tabular}{|c|c|c|c|c|c|c|c|}
\hline \multirow{2}{*}{ Scoring } & \multirow{2}{*}{ with Category } & \multicolumn{2}{|c|}{ AT First Follow up } & \multicolumn{2}{|c|}{ At second Follow up } & \multicolumn{2}{|c|}{ At Third Follow up } \\
\hline & & $\mathbf{n}$ & $\%$ & $\mathbf{n}$ & $\%$ & $\mathbf{n}$ & $\%$ \\
\hline$<55$ & Poor & 32 & 86.5 & 10 & 27.0 & 2 & 5.4 \\
\hline $55-69$ & Fair & 4 & 10.8 & 12 & 32.4 & 3 & 8.1 \\
\hline $70-85$ & Good & 1 & 2.7 & 15 & 40.5 & 18 & 48.6 \\
\hline$>85$ & Excellent & 0 & 0.0 & 0 & 0.0 & 14 & 37.8 \\
\hline Total & & 37 & 100.0 & 37 & 100.0 & 37 & 100.0 \\
\hline \multicolumn{2}{|c|}{ Mean \pm Std. Deviation } & \multicolumn{2}{|c|}{$43.54 \pm 10.65$ points } & \multicolumn{2}{|c|}{$63.11 \pm 12.50$ points } & \multicolumn{2}{|c|}{$79.03 \pm 12.15$ points } \\
\hline
\end{tabular}




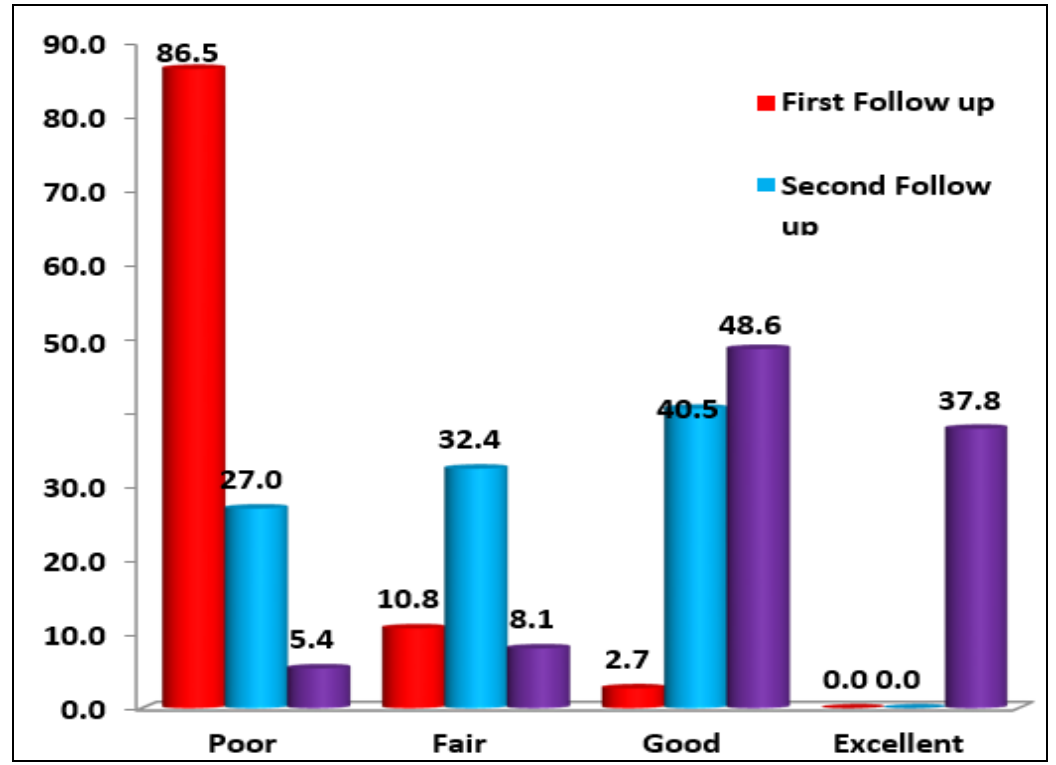

Fig 5: Distribution of clinical and functional outcome at first, second and third follow ups

Table 5: Distribution of Time of Union of Distal Femur Fracture Patients

\begin{tabular}{|c|c|c|}
\hline Time of union & Frequency (N) & Percent (\%) \\
\hline $11-13$ weeks & 7 & 18.9 \\
\hline $13-15$ weeks & 11 & 29.7 \\
\hline $15-17$ weeks & 11 & 29.7 \\
\hline $17-19$ weeks & 8 & 21.6 \\
\hline Total & 37 & 100 \\
\hline
\end{tabular}

Most of the cases (44\%) attained radiological union in 19-22 weeks. The mean time for radiological union was 19 weeks

\section{Discussion}

The mean age in our study was 45.70years. A study published by Reddy J.A.V mean age was 40 years (range 20-70) [23] Ramanand M. et al found mean age 52.25 years (range 20-60 years) ${ }^{[24]}$. Dr. Pugazhendhi et al. conducted a short term prospective study of the functional outcome in distal femoral fractures treated by locking compression plate, they reported that out of 22 patients, there were 12 patients in 21-30 age group, 5 patients in 31-40 age group, 3 patients in 41-50 age group and 2 patients in 51-60 age group. They concluded that the most common age group was 21-30 years ${ }^{[25]}$. Scope of distal femur locking plate is limited not only to isolated distal femur fractures but also in the use of peri-prosthetic distal femur fractures in patients of Total Hip Replacement (THR) ${ }^{[26]}$ and Total Knee Replacement (TKR). Even extreme distal peri-prosthetic supracondylar fractures can be managed with lateral locked plate with predictable results similar to those seen in more proximal fractures ${ }^{[27]}$.

In our study, the average time of union was 14.5 weeks, with majority of cases united between 13-17 weeks. Time of union in other studies are tabulated below in table

Time of union in other studies are tabulated below in table

\begin{tabular}{|c|c|}
\hline Author & Time of union (weeks) \\
\hline Shreedhar M. ${\text { et } a l^{[28]}}^{[2]}$ & 20 \\
\hline Pugazhendhi G. et $_{\text {al }}{ }^{25]}$ & 16 \\
\hline Reddy J.A.V. et al $^{[23]}$ & 12 \\
\hline Shriharsha et $_{\text {al }}{ }^{[29]}$ & 19.6 \\
\hline Panchal P. et al $^{[30]}$ & 18 \\
\hline Ramesh Krishna et $^{\text {al }}{ }^{[1]}$ & 15.3 \\
\hline Mahesh D.V. et al $^{[10]}$ & 18 \\
\hline Our study & 14.3 \\
\hline
\end{tabular}

In our study, by using Neer's society scoring system, the results were excellent in $14(37.8 \%)$, good in $18(48.6 \%)$, fair in $3(8.1 \%)$ and poor in $2(5.4 \%)$ patients. Our study was comparable with standard studies of management of distal femur fracture using distal femoral locking compression plate. Functional outcome of other studies are tabulated below in table

Functional outcome of other studies are tabulated below in table

\begin{tabular}{|c|c|c|c|c|}
\hline Author & \multicolumn{4}{|c|}{ Results } \\
\hline & Excellent & Good & Fair & Poor \\
\hline Ramesh Krishna et al. ${ }^{[1]}$ & 15 & 11 & 3 & 1 \\
\hline Tapi Nalo et al. ${ }^{[31]}$ & 25 & 3 & 1 & 0 \\
\hline C.V. Dasaraiah et al. ${ }^{[2]}$ & 15 & 11 & 3 & 1 \\
\hline Pradip B Patil et al. ${ }^{[32]}$ & 18 & 11 & 1 & 0 \\
\hline Shriharsha R.V. et al. ${ }^{[29]}$ & 4 & 10 & 5 & 6 \\
\hline Parth Panchal et al. ${ }^{[30]}$ & 11 & 5 & 3 & 1 \\
\hline Our study & 14 & 18 & 3 & 2 \\
\hline
\end{tabular}

Dr. Pugazhendhi G. et al. Concluded that, the early complications were encountered in 3 patients, 2 patients developed superficial infection and 1 patient had wound gaping. Late complications included knee stiffness in2 patients. None of the patients had loss of fixation or malunion or non

Union [25] According to Parth Panchal [30] et al study on treatment of distal end of fracture femur by locking compression plate, 5 patients developed complications, with 4 (20\%) patients experiencing superficial infection and $1(5 \%)$ patients delayed union. Tapi Nalo et al conducted the study on treatment of supracondylar fracture of distal femur with condylar locking compression plating, and they reported that, there were 2 cases of superficial infection ${ }^{[31]}$.

Limitation: The requirement for more number of patients was felt during the conduct of our study but with a longer follow-up of 2 years it was reasonable to restrict ourselves to a follow-up compliant group.

\section{Conclusion}

Distal femur locking plate is still the way forward for treating distal femur fractures. Positive results have been published by researchers with implants such as distal femur nail, dynamic condylar screw and even addition of a medial plate to a distal 
femur locking plate for treating distal femur fractures. By conducting this study we can put forward the assumption with some degree of confidence that fractures of the distal femur of all varieties extra-articular, partial articular and intra-articular non-comminuted as well as comminuted ones, if fixed in a proper fashion following all the basic principles of fracture fixation, good results can be obtained by using a distal femurlocking plate alone as the main implant of choice for such fractures. It has also reduced the requirement of secondary procedures such as bone grafting especially in fractures with metaphyseal comminution.

\section{Reference}

1. Krishna RK, Nayak MA. Study of surgical management of distal femoral fracture by distal femoral locking compression plateosteosynthesis. IJOS 2015;1(1):22-6.

2. Dasaraiah CV, Rao AS. Study of surgical management of supracondylar femoral fracture by locking compression plate. IOSR Journal of Dental and Medical Sciences (IOSR-JDMS) 2016;15(2):23-33.

3. Martinet O, Cordey J, Harder Y, Maier A, Buhler M, Barraud GE. Epidemiology of fracture of distal femur. Injury 2000;31:62-63

4. Arneson TJ, Melton LJ III, Lewallen DG, O'Fallon WM. Epidemiology of diaphyseal and distal femoral fractures in Rochester, Minnesota, 1965-1984. Clin Orthop 1988;234:188-94

5. Gupta SKV, Govindappa CVS, Yalamanchili RK. Outcome of retrograde intramedullary nailing and locking compression plating of distal femoral fractures in adults. Open access Orthopaedics 2013;1(3):23.

6. Crist B, Rocca G, Murtha Y. Treatment of acute distal femur fractures. Orthopaedics 2008;31:681.

7. Higgins TF. "Distal femoral fractures," The Journal of Knee Surgery 2007;20(1):56-66.

8. Hakeem A, Khan N, Khan M, Ullah F. Dynamic condylar screw fixation in treatment of supracondylar fracture of distal femur. Rawal medical journal 2010;35(1):45-47.

9. Mahesh DV, Gunnaiah, Vishwanath. Management of distal femur fracture by locking compression plate. IJHSR. 2014;4(5):235-40.

10. Yeap EJ, Deepak AS. Distal femoral locking compression plate fixation in distal femoral fractures: early results. Malaysian Orthopaedic Journal 2007;1(1):12-7.

11. Schatzker J, Lambert DC. Supracondylar fractures of the femur.Clin Orthop 1979;138:77-83.

12. Wilson JN. Watson Jone's: Fractures and joint injuries. 6thed 1982, 1003-070,

13. Neer CSII, Grantham SA, Shelton ML. Supracondylar Fracture of the Adult Femur - A Study of One Hundred and Ten Cases. JBJS Am 1967;49-A(4):591-613.

14. Brown A, D'Arcy JC. Internal Fixation for Supracondylar Fractures of the Femur in the Elderly patient. JBJS 1971;53B(3):420-424.

15. Thomas P Reudi, Richard E Buckley, Christopher G Moran. A O Principles of Fracture Management. 2nd edition. Switzerland: AO Publishing 2007.

16. Krettek C, Schandelmaier P, Miclau T, Tscherne H. Minimally Invasive Percutaneous Plate Osteosynthesis (MIPPO) using the DCS in Proximal and Distal Femoral Fractures. Injury 1997;28(1):20-30.

17. Kregor PJ, Stannard J, Zlowodzki M, Cole PA, Alonso J. Distal femoral fracture fixation utilizing the Less Invasive Stabilization System (L.I.S.S.): The technique and early results. Injury 2001;32(3):32-47.

18. 19 Smith WR, Ziran BH, Anglen JO, Stahel PF. Locking plates: tips and tricks. J Bone Joint Surgery Am. 2007;89(10):2298-307.

19. Venable CS, Stuck WG, Beach A. The Effects on Bone of the Presence of Metals: Based on electrolysis. Ann Surg 1937.

20. Kregor PJ, Stannard JA, Zlowodzki M, Cole PA. Treatment of Distal Femur Fractures Using the Less Invasive Stabilization System: Surgical Experience and Early Clinical Results in 103 Fractures. J Orthop Trauma 2004;18(8):509-20.

21. Kumar GNK, Sharma G, Farooque K, Sharma V, Ratan $\mathrm{R}$, Yadav S, Lakhotia D. Locking compression plate in distal femoral intraarticular fractures: our experience. International Scholarly Research Notices Article ID 372916, 2014, 5.

22. Reddy JAV, Chary NB. Functional outcome of distal end of femur with locking compression plate: a prospective study. Ann. Int. Med. Den. Res 2016;2(1):362-65.

23. Ramanand M, Mudgal CV, Nagendrappa M. Management of distal femoral fractures- A comparative study between supracondylar nail and distal femoral locking plate. J Evid. Based Med. Healthc. 2017;4(34):2073-2077.

24. Pugazhendhi G, Venkatachalam K, Madhukar. A short term prospective study of the functional outcome in distal femoral fractures treated by locking compression condylar plates. Sch. Acad. J Biosci 2016; 4(9):707-11

25. Chakravarthy J, Bansal R, Cooper J. Locking plate osteosynthesis for Vancouver Type B1 and Type C periprosthetic fractures of femur: a report on 12 patients. Injury 2007;38(6):725-33

26. Streubel PN, Gardner MJ, Morshed S, Collinge CA, Gallagher B, Ricci WM et al. Are extreme distal periprosthetic supracondylar fractures of the femur too distal to fix using a lateral locked plate? J Bone Joint Surg Br 2010;92(4):527-34.

27. Sreedhar M, Luther ME, Anudeep B, Prashanth, Swaroopa, Madhav V. Functional and radiological outcome of distal femoral fractures treated with locking compression plate. Journal of Evolution of Medical and Dental Sciences 2015;4(38):6664-71.

28. Shriharsha RV, Sapna M. Utility and outcomes of locking compression plates in distal femoral fractures. Int J Res Orthop 2015;1(1):15-21

29. Panchal P, Patel C, Poptani A. Treatment of distal end of fracture femur by lockin compression plate; Int. J Med Science and Pub Health 2016;5;09

30. Nalo T, Agrahari A, Datta S, Paul V, Singh SN, Langshong R. Treatment of Supracondylar Fracture of Distal Femur with Condylar Locking Compression Plating. International Journal of Science and Research (IJSR) ISSN (Online): 2319-7064

31. Patil PB, Patil RS, Lad SA, Suren T, Jain UD. The study of functional outcome of distal end femur fractures operated with locking compression plate; Asian Pac J Health Sci 2016;3(1):135-139. 